\title{
TENDÊNCIA DO AUMENTO DE CHUVAS E SUAS IMPLICAÇÕES NA ESTABILIDADE DE ENCOSTAS NO SUL DE SANTA CATARINA
}

\section{TREND OF INCREASING RAIN AND THEIR IMPLICATIONS ON THE STABILITY OF SLOPES IN SOUTH SANTA CATARINA}

\section{William de Oliveira Sant Ana \\ Doutorando do PPGCA/UNESC. E-mail: williamsantana@gmail.co $\mathrm{m}$}

\section{Álvaro José Back}

Professor Dr. do PPGCA/UNESC. E-mail: ajb@unesc.ne

\section{RESUMO}

Extremos climáticos vêm sendo registrados frequentemente no Brasil e impactam no cotidiano econômico-ambiental. No Estado de Santa Catarina, o aumento dos totais de chuva implica diretamente em escorregamentos de encostas, inundações, perdas agrícolas, dentre outros problemas ambientais e urbanos ou até, perda de vidas. O objetivo deste estudo foi identificar, com análise estatística, por meio de Regressão Linear e do teste Mann-Kendall, possíveis tendências ao aumento de chuvas para o sul catarinense. Os dados foram fornecidos pela estação meteorológica INMET-289, localizada na cidade de Urussanga, datados desde 01 de janeiro de 1950 até 31 de dezembro de 2016. Foram realizadas as análises de Regressão Linear e de Mann-Kendall, para cada série mensal. Como resultados, existem alguns meses em que há tendência de aumento de precipitação pluviométrica para o sul catarinense, com destaque para o quarto trimestre do ano e mesmo para as chuvas anuais. Observou-se no teste Mann-Kendall que, para a série anual, a tendência é significativa $(\mathrm{p}=0,0002)$, sendo a magnitude de $6,675 \mathrm{~mm}$ ano $^{-1}$, o que corresponde a aumento de $66,7 \mathrm{~mm}$ de chuva por década. De fato, como haverá mais chuva para o sul catarinense, um solo saturado irá apresentar maior tensão cisalhante, ficando sujeito a escorregamentos.

Palavras-chave: Mudanças climáticas. Aumento de precipitação. Desastres.

\begin{abstract}
Climatic extremes have been frequently registred in Brazil and impact on the economic and environmental everyday. In the state of Santa Catarina, the increase in rainfall totals directly implies landslides, floods, agricultural losses, among other environmental and urban problems or even loss of life. The objective of this study was to identify, with statistical analysis, as Linear Regression and the MannKendall test, possible trends for increasing rainfall for southern Santa Catarina. Data were provided by weather station INMET-289, located in the city of Urussanga, intevaled January 1, 1950 to December 31, 2016. Linear Regression and Mann-Kendall analyzes were performed for each monthly series. As a result, there are some months in which there is a trend of increasing rainfall for the south of Santa Catarina, with emphasis on the fourth quarter of the year and even the annual rainfall. It was observed in the Mann-Kendall test that, for the annual series, the trend is significant $(\mathrm{p}=0.0002)$, with a magnitude of $6.675 \mathrm{~mm}$ year-1, which corresponds to an increase of $66.7 \mathrm{~mm}$ of rainfall per decade. In fact, as there will be more rain to the south of Santa Catarina, a saturated soil will present higher shear stress, being subject to landslides.
\end{abstract}

Keywords: Climate change. Increased precipitation. Disasters. 


\section{INTRODUÇÃO}

O ano de 1988 marcou a data de fundação do Painel Intergovernamental de Mudanças Climáticas (IPCC, na sigla em inglês), e, com o aquecimento planetário, a acentuação de desvios climáticos passou a ser assunto de interesse científico. No quinto relatório (IPCC AR5), disponibilizado em 2013 e 2014 (IPCC, 2013; IPCC, 2014), com 95\% de certeza, foi afirmado que há aumento de temperatura na ordem de $0,9^{\circ} \mathrm{C}$, com base no ano de 1850 , e que nos últimos 50 anos a causa principal é antrópica, por meio da emissão de gases estufa (MARENGO, 2014).

De acordo com Denski e Back (2015), essas alterações climáticas mundiais têm gerado preocupação, levando-se em conta que, se as emissões de gases estufa se mantiverem nas taxas atuais, até o final do século haverá um aumento de até $4,8^{\circ} \mathrm{C}$ na temperatura média global. Yu et al. (2002) já alertavam que a manutenção de tendências altera elementos do clima, como temperatura, umidade e chuvas, não só na escala planetária, mas também regional.

Os extremos climáticos que vêm sendo registrados frequentemente no Brasil impactaram diretamente o cotidiano econômico-ambiental, a exemplo do desabastecimento hídrico, planejamento de obras hidráulicas, de saneamento urbano (galerias pluviais, por exemplo) e até mesmo do comprometimento da segurança energética. Existem evidências científicas suficientes de que o clima futuro, no Brasil, será mais quente e chuvoso e que as atividades antrópicas podem ter significativa participação nesse processo de mudanças climáticas (MARENGO, 2014).

Num volume especial que analisa extremos climáticos, o SREX (IPCC, 2012) enfatiza que, nos últimos 30 anos, aumentou a irregularidade de chuvas na América do Sul, com extremos de chuvas intensas. De acordo com Marengo (2014), observa-se que, no Brasil, está havendo acentuação de chuvas no verão, bem como aumento de frequência de chuvas fortes no inverno, isso para o Sul e o Sudeste. A previsão executada por modelos rodados em supercomputadores sugere tendências de aumento de chuva nessas regiões, e, se aumenta a disponibilidade hídrica, mesmo que irregularmente distribuída, incertezas adicionais redundam, como de que forma proceder nas medidas de adaptação e mitigação.

No Sul do Brasil, particularmente no Estado de Santa Catarina, aumento dos totais e dos picos de chuva implicam diretamente em escorregamentos de encostas, inundações, perdas agrícolas, deflagração de problemas ambientais urbanos, veiculação de doenças e até a perda de vidas. Esses eventos implicam em grandes prejuízos econômicos e altos custos sociais, particularmente para o estado de Santa Catarina. Os custos totais das inundações e movimentos de massa de novembro de 2008 (concentrado em municípios do Vale do Itajaí, como Blumenau e Ilhota) foram calculados pelo 
Banco Mundial em R \$ 4,75 bilhões, o que na época representava 2,67\% do PIB do Estado (CEPEDUFSC, 2013).

Diante dessa problemática, analisar o comportamento das chuvas é de grande importância para o entendimento da dinâmica ambiental, contribuindo para antever reflexos nos setores produtivos da sociedade e para o planejamento e resiliência dessa. Alguns estudos realizados sobre séries de precipitação da região Sul do Brasil identificaram tendências (HAYLOCK et al., 2006). Campos et al. (2006) encontraram um aumento dos totais anuais de precipitação no período entre 1911 e 2006 no Estado de Santa Catarina. Minuzzi e Caramori (2011) estudaram dados de 21 estações pluviométricas do Paraná, em que observaram aumento da quantidade de chuva, principalmente no verão. Pinheiro et al. (2013), analisando dados do Sul do Brasil, observaram aumento de chuvas em 17 das 18 estações analisadas.

Para o Sul de Santa Catarina, Pezente (2017) realizou uma análise temporal do comportamento de chuvas anuais, entre 1950 e 2016, procurando classificar os anos em secos, chuvosos ou normais. Denski e Back (2015) procuraram averiguar tendências de elementos climatológicos com foco na evapotranspiração. Em comum, ambos os estudos realizados se utilizaram de dados fornecidos pela Empresa de Pesquisa e Extensão Rural de Santa Catarina (EPAGRI) e pelo Instituto Nacional de Meteorologia (INMET), que operam estações no município de Urussanga. De fato, essa possui a série mais longa e confiável para estudos do clima sul catarinense.

O objetivo deste estudo foi identificar, com a análise estatística, por meio do teste MannKendall e modelo de Regressão Linear e seus respectivos testes de validade de modelo, possíveis tendências ao aumento de chuvas mensais para o sul catarinense, utilizando-se dos dados mensais de chuvas do período de 1950 a 2016, da estação meteorológica de Urussanga (289 - INMET) (Figura $1)$.

\subsection{CLIMA LOCAL}

De acordo com o sistema de classificação global dos tipos climáticos, proposto por KöppenGeiger (AYOADE, 1996), a conjunção da atuação sazonal das diferentes massas de ar, acrescida de fatores, como latitude, altitude e maritimidade/continentalidade, condiciona o sul catarinense ao clima temperado chuvoso, úmido em todas as estações, com verão quente (Cfa). Conforme Sônego (2002, p. 102), no sul catarinense predomina o clima de latitudes subtropicais:

[...] O sul de Santa Catarina apresenta clima quente no verão e ameno no inverno, com chuvas bem distribuídas durante o ano. O clima é Subtropical Úmido com Verão Quente, pelo sistema de Köppen, recebendo a simbologia Cfa. A temperatura média anual varia de 17 a $20^{\circ} \mathrm{C}$, com a temperatura média das máximas variando de 23 a $27^{\circ} \mathrm{C}$, e a média das mínimas variando de 13 a $17^{\circ} \mathrm{C}$. A altitude e a sua distância com o Oceano Atlântico são os fatores 
que determinam essas variações espaciais de temperatura, já que existe pouca variação de latitude dentro da região.

Figura 1 - Localização de urussanga no sul de Santa Catarina e da estação meteorológica INMET 289

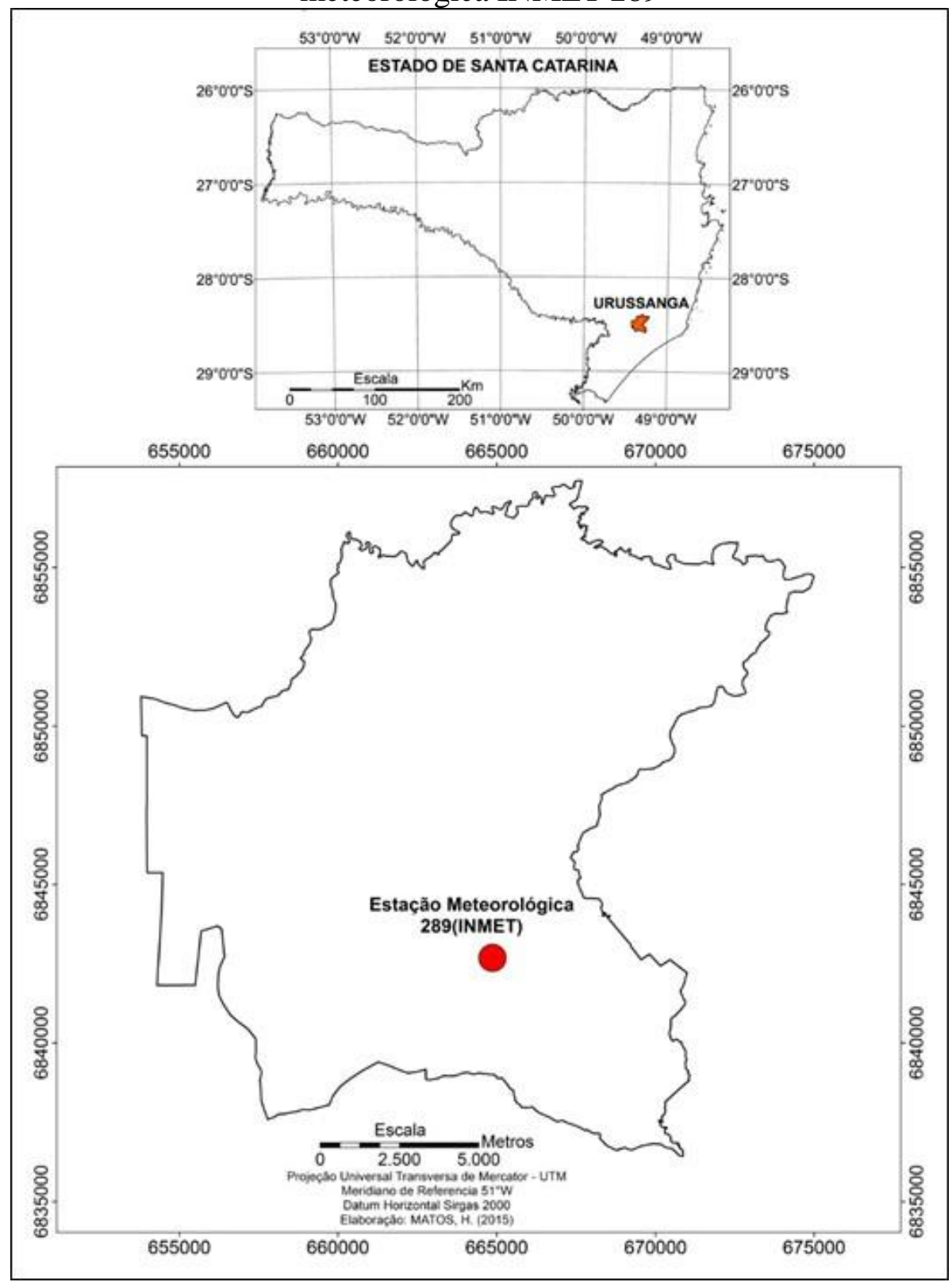

Fonte: Denski e Back (2015)

Segundo Monteiro e Furtado (1996), predominam no Estado de Santa Catarina, durante o verão, massas de ar com centros de origens tropicais e equatoriais, ou seja, com essas características, a exemplo das massas de ar Equatorial Continental (mEc), Tropical Atlântica (mTa) e, eventualmente, Tropical Continental (mTc). No inverno, a passagem da Frente Polar Atlântica (FPA) precede à chegada da Massa Polar Atlântica (mPa), que, respectivamente, ocasionam chuvas frontais e declínio da temperatura e da umidade, deslocando as massas tropicais para o centro e o norte do país. 
Menciona-se, também, a influência da orografia na formação e distribuição de chuvas, mais comum entre os meses de setembro e março, tendo em vista a circulação atmosférica de ventos de direção nordeste, úmidos, alimentados pela Massa Tropical Atlântica que está posicionada sobre o Atlântico Sul. Esses ventos se defrontam com a escarpa da Serra Geral e formam chuvas orográficas. Devem também ser registradas as chuvas convectivas, típicas dos meses mais quentes do ano.

De acordo com Sônego (2002, p. 102), o sul catarinense apresenta maior precipitação quando perto da Serra Geral:

[...] os maiores valores são observados nas encostas da Serra Geral, diferença esta causada pelas chuvas de verão que são mais frequentes e intensas nas encostas da Serra Geral do que na orla. Isto se deve ao fato de que, ao encontrar a Serra Geral, o ar úmido é forçado a se elevar causando a formação de nuvens geradoras de temporais de final de tarde de verão.

Pelo observado, as chuvas locais já se apresentam com desvios positivos na região, dado ao fator orográfico e convectivo dos meses mais quentes, ou seja, quaisquer tendências de aumento da precipitação pluviométrica impactariam diretamente as relações socioeconômico-ambientais que estão fortemente atreladas ao ciclo hidrológico. Complementa-se que, tangenciando todo o sul catarinense, há a escarpa de bordo de Planalto Sul Brasileiro, uma área de elevada altimetria e declividades, favorável para escorregamentos.

\section{MATERIAIS E MÉTODOS}

Este trabalho foi desenvolvido a partir de dados de chuva datados desde 01 de janeiro de 1950 até 31 de dezembro de 2016, organizados por totais mensais. Os dados foram fornecidos pela estação meteorológica do Instituto Nacional de Meteorologia, INMET 289. A estação está localizada sob as coordenadas $28^{\circ} 31^{\prime} 00^{\prime \prime} \mathrm{S}$ e $49^{\circ} 19^{\prime} 00^{\prime \prime} \mathrm{W}$, com altitude de 48,17 metros no município de Urussanga, SC, conforme já apresentado na Figura 1.

Adicionalmente, para entender a relação do aumento de chuvas no comportamento de encostas, foram elencados trabalhos que abordaram a relação entre florestas (KOBIYAMA et al., 2012), ciclo hidrológico (HONDA; DURIGAN, 2017), efeito da vegetação na estabilidade geral das encostas (MICHEL, 2013), além de raízes e estabilização de encostas (REUBENS et al., 2007).

Os dados de chuva foram organizados em planilha no software Excel ${ }^{\circledR}$ e por meio do menu Análise de Dados e do software ActionStat ${ }^{\circledR}$ (PORTAL ACTION, 2018), que utiliza a plataforma R, principal linguagem de programação estatística de uso mundial. Foram realizadas as Análises de Regressão Linear e de Mann-Kendall, para cada série mensal. Back (2001) já havia empregado análise estatística como método para identificar tendências climáticas, e Back e Vieira (2009), para verificar tendências de chuva na estação meteorológica de Urussanga. 


\subsection{ANÁLISE DE REGRESSÃO LINEAR}

A análise de regressão é um conjunto de técnicas estatísticas que tratam da formulação de modelos estatísticos que especificam relações entre variáveis, e do uso desses modelos para propósitos de inferências, particularmente predição. Conforme Selinus e Esbensen (1995), um dos métodos estatísticos mais empregados no estudo de parâmetros ambientais e que trata da situação de uma única variável resposta é a análise de regressão linear.

A análise de regressão linear simples compreende um conjunto de procedimentos para a derivação de inferências estatísticas referentes à relação entre uma variável resposta Y e uma variável explanatória X:

$\mathbf{E}(\mathbf{Y})=\mathbf{a}+\beta \mathbf{X}$

Em que b é denominado coeficiente angular ou inclinação da reta, e representa a tendência da série temporal.

Neste estudo, a variável resposta que se procura é a predição de chuvas, com base na série histórica mensal que serve de explanação. Como teste de validade do modelo, será analisada a variância pela distribuição Fischer-Snedecor, em que se assume "F de Significação", ou valor de "p", menor ou igual a 0,05 para um modelo válido. Não serão realizadas análise de resíduos.

\subsection{ANÁLISE DE MANN-KENDALL}

A análise de Mann-Kendall consiste num teste não paramétrico útil para detectar tendências de um conjunto de dados, supondo-se que sejam observações independentes, sendo as observações $\mathrm{X}_{1}, \mathrm{X}_{2} \ldots \mathrm{X}_{\mathrm{n}}$ de uma série temporal. $\mathrm{O}$ objetivo de sua aplicação consiste em testar as observações da série, sua independência e distribuição, ou seja, testar as hipóteses $\mathrm{H}_{0}$ (não há tendência), ou $\mathrm{H}_{1}$, em que há tendência monotônica no tempo (PORTAL ACTION, 2018).

De acordo com Denski e Back (2015), esse teste foi proposto em 1975 por Sneyers, sendo que o teste considera que a sucessão de valores ocorrerá de forma independente e a distribuição de probabilidade permanecerá sempre a mesma numa série aleatória simples, isso na hipótese de estabilidade de uma série temporal. Segundo Goossens e Berger (1986), esse teste é apropriado para se realizar análise de séries climatológicas, inclusive, oportunizando averiguar o momento aproximado inicial de uma tendência.

As hipóteses a serem testadas são: 
a) Ho. Os dados são uma amostra de uma variável aleatória de $\mathrm{n}$ dados independentes e identicamente distribuídos (isto é, não existe tendência no conjunto de dados);

b) $\quad H_{1}$. A distribuição dos dados xi e xj não é idêntica para todos $i, j \leq N$, em que $i \neq$ onde i e $j$ são as sequência dos dados e $\mathrm{N}$ é o comprimento da série.

Para o teste bicaudal, ao nível de significância $\alpha$, rejeita-se $\mathrm{H}_{0}$ se o teste estatístico padronizado, $\mathrm{Z}<\mathrm{Z}_{\alpha / 2}$ ou $\mathrm{Z}>\mathrm{Z}_{\alpha / 2}$, em que a estatística normalizada de Mann-Kendall segue a distribuição normal com média zero $(0,0)$ e variância um $(1,0)$.

$Z=\left\{\begin{array}{c}\frac{s-1}{\sqrt{\operatorname{var}(S)}} \text { se } S>0 \\ 0 \text { se } S=0 \\ \frac{s+1}{\sqrt{\operatorname{var}(S)}} \text { se } S<0\end{array}\right\}$

A estatística Mann-Kendall (S) é dada por:

$S=\sum_{j=1}^{N-1} \sum_{i=j+1}^{N} \operatorname{sgn}\left(x_{i}-x_{j}\right)$

Em que:

$\operatorname{sgn}(\theta)=\left\{\begin{array}{c}1 \text { se } \theta>0 \\ 0 \text { se } \theta=0 \\ -1 \text { se } \theta<0\end{array}\right\}$

Para variável aleatória independente distribuída identicamente (sem tendência), a média a e variância de S são dadas por:

$\mathrm{E}(\mathrm{S})=0$

$\operatorname{Var}(S)=\frac{N(N-1)(2 N+5)-\sum_{t}^{N} t(t-1)(2 t+5)}{18}$

Em que $\mathrm{t}=$ extensão a qualquer empate.

Há dois importantes parâmetros no teste de tendência. O nível de significância local, também chamado de p-valor, indicando a quantidade de evidência para rejeitar $\mathrm{H}_{0}$. Outra característica da 
tendência de interesse é a magnitude e a direção da tendência. O teste de Mann-Kendall não fornece estimativa da magnitude da tendência. Para obter essa estimativa, foi utilizado o método descrito por Helsel e Hirsch (1991), com o teste não paramétrico de Theil-Sen (HELSEL; HIRSCH, 2002), para obter a estimativa robusta da inclinação $(\beta)$ indicando a magnitude e a tendência da declividade.

A estimativa da inclinação ( $\beta$ ) é definida como:

$\boldsymbol{\beta}=\operatorname{mediana}\left(\frac{Y_{j}-Y_{i}}{t_{j}-t_{i}}\right)$, para todos $\mathrm{i}<\mathrm{j}$

\section{RESULTADOS}

\subsection{ANÁLISES DE REGRESSÃO PARA CHUVAS MENSAIS}

A Análise de Regressão Linear para os dados mensais de chuva, no intervalo de 1950 a 2016 na estação meteorológica de Urussanga, bem como análise dos totais anuais desse período, pode ser visualizada na Tabela 1. Já a análise particionada por trimestres apresenta-se na Tabela 2.

Observa-se que em todos os meses o valor da inclinação é positiva, o que indica tendências de aumento na precipitação. No entanto, somente nas séries dos meses de maio, dezembro e anual, a tendência foi estatisticamente significativa $(\mathrm{p}<0,05)$. Para as demais séries, com $\mathrm{p}>0,05$, não se pode rejeitar a hipótese de que não existem tendências nessas séries, no entanto não pode ser interpretado como afirmação de que as séries são estacionárias. Somente não há evidências suficientemente fortes para rejeitar a hipótese $\mathrm{H}_{0}$ ao nível de $5 \%$ de significância.

Tabela 1 - análise de regressão linear dos dados de chuvas (1950-2016)

\begin{tabular}{c|r|r|r|r}
\hline & \multicolumn{1}{|c|}{$\mathrm{b}$} & \multicolumn{1}{c|}{ Lim Inferior } & \multicolumn{1}{c}{ Lim Superior } & \multicolumn{1}{c}{ p- valor } \\
\hline Jan & 1,1629 & $-0,0711$ & 2,3968 & 0,0643 \\
\hline Fev & 0,5639 & $-0,6096$ & 1,7373 & 0,3408 \\
\hline Mar & 0,0624 & $-1,1448$ & 1,2695 & 0,9181 \\
\hline Abr & 0,0312 & $-0,6364$ & 0,6989 & 0,9259 \\
\hline Maio & 0,988 & 0,0077 & 1,97 & 0,048 \\
\hline Jun & 0,3694 & $-0,2271$ & 0,966 & 0,2206 \\
\hline Jul & 0,729 & $-0,0627$ & 1,5207 & 0,0705 \\
\hline Ago & 0,3875 & $-0,8197$ & 1,5947 & 0,5238 \\
\hline Set & 0,2443 & $-0,6962$ & 1,1848 & 0,6056 \\
\hline Out & 0,4925 & $-0,2826$ & 1,2675 & 0,209 \\
\hline Nov & 0,5445 & $-0,2528$ & 1,3418 & 0,1773 \\
\hline Dez & 1,094 & 0,079 & 2,109 & 0,035 \\
\hline Ano & 6,6705 & 3,298 & 10,0431 & 0,0002 \\
\hline
\end{tabular}

Fonte: Elaborada pelos autores (2018).

Quando se agrupa os meses por trimestres, o $2 .^{\circ}$ trimestre, dada influência de maio, aproximou-se de apresentar tendência, registrando " $p$ " de 0,0501, contudo, com 0,0119, o trimestre que reúne outubro, novembro e dezembro é o único em que a regressão acusa tendência. Dessa forma, 
considera-se que, pela análise de regressão, existem alguns meses em que há tendência de aumento de precipitação pluviométrica para o sul catarinense, com destaque para o quarto trimestre do ano, e mesmo para as chuvas anuais.

Tabela 2 - Análise de regressão linear organizada trimestralmente

\begin{tabular}{c|r|r|r|r}
\hline & Inclinação b & Lim Inferior & Lim Superior & \multicolumn{1}{c}{ p- valor } \\
\hline $1^{\circ}$ trimestre & 1,7891 & $-0,287$ & 3,8647 & 0,0899 \\
\hline $2^{\circ}$ trimestre & 1,3895 & $-0,0005$ & 2,7796 & 0,0501 \\
\hline $3^{\circ}$ trimestre & 1,3608 & $-0,4176$ & 3,1392 & 0,1313 \\
\hline $4^{\circ}$ trimestre & 2,1311 & 0,4871 & 3,7751 & 0,0119 \\
\hline
\end{tabular}

Fonte: Elaborada pelos autores (2018).

Como forma de representação dos valores absolutos de chuvas nos meses de tendência positiva (maio e dezembro) sua comparação com os dados de normal do período (1950-2016), acompanhamento de tendência linear e da equação da reta (que representa o modelo de regressão linear) podem ser acompanhadas as Figuras 2 e 3.

Figura 2 - Chuvas de maio (1950-2016) e comparativo com a normal do mês e tendência

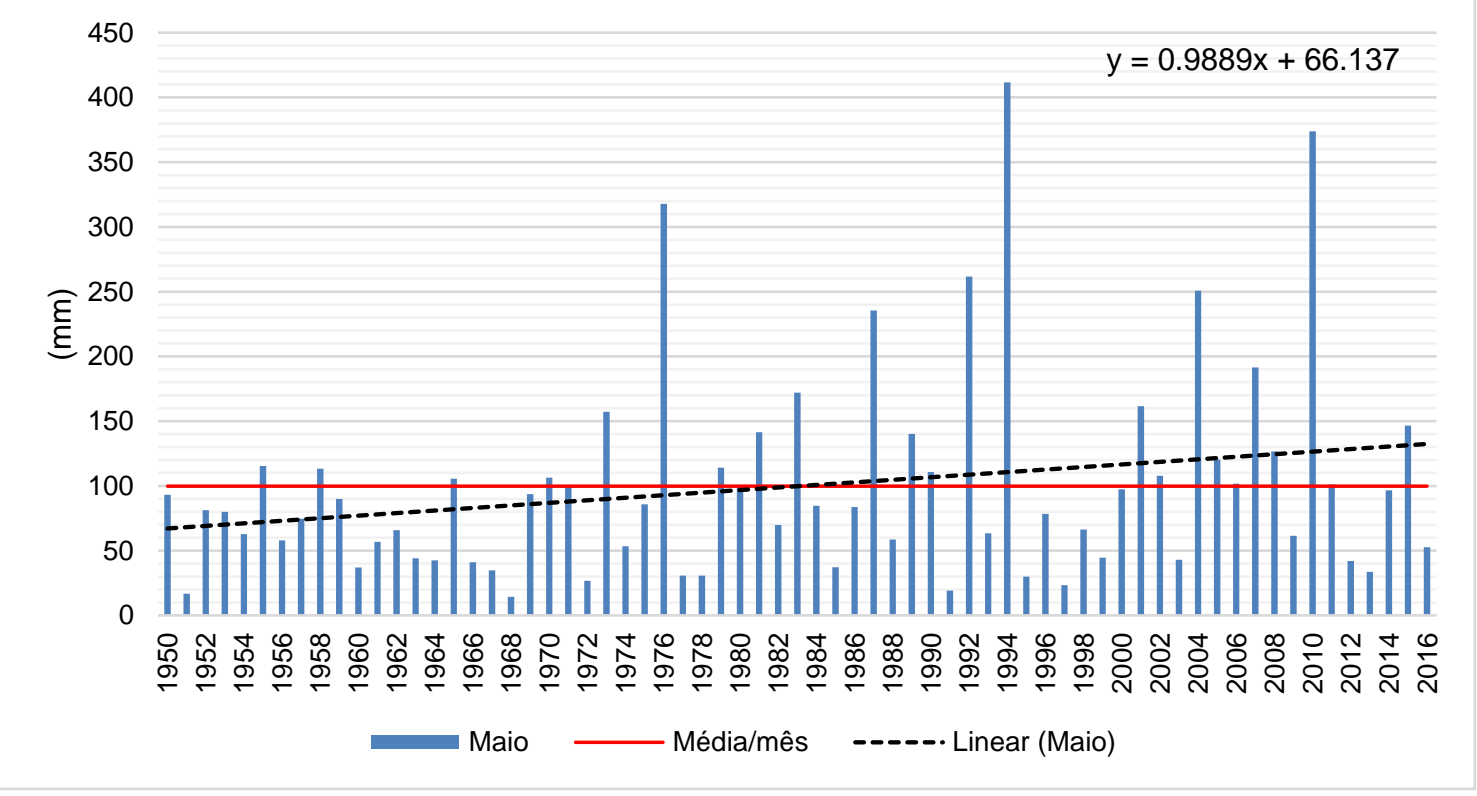

Fonte: Elaborado pelos autores (2018).

Figura 3 - Chuvas de dezembro (1950-2016) e comparativo com normal do mês e tendência 


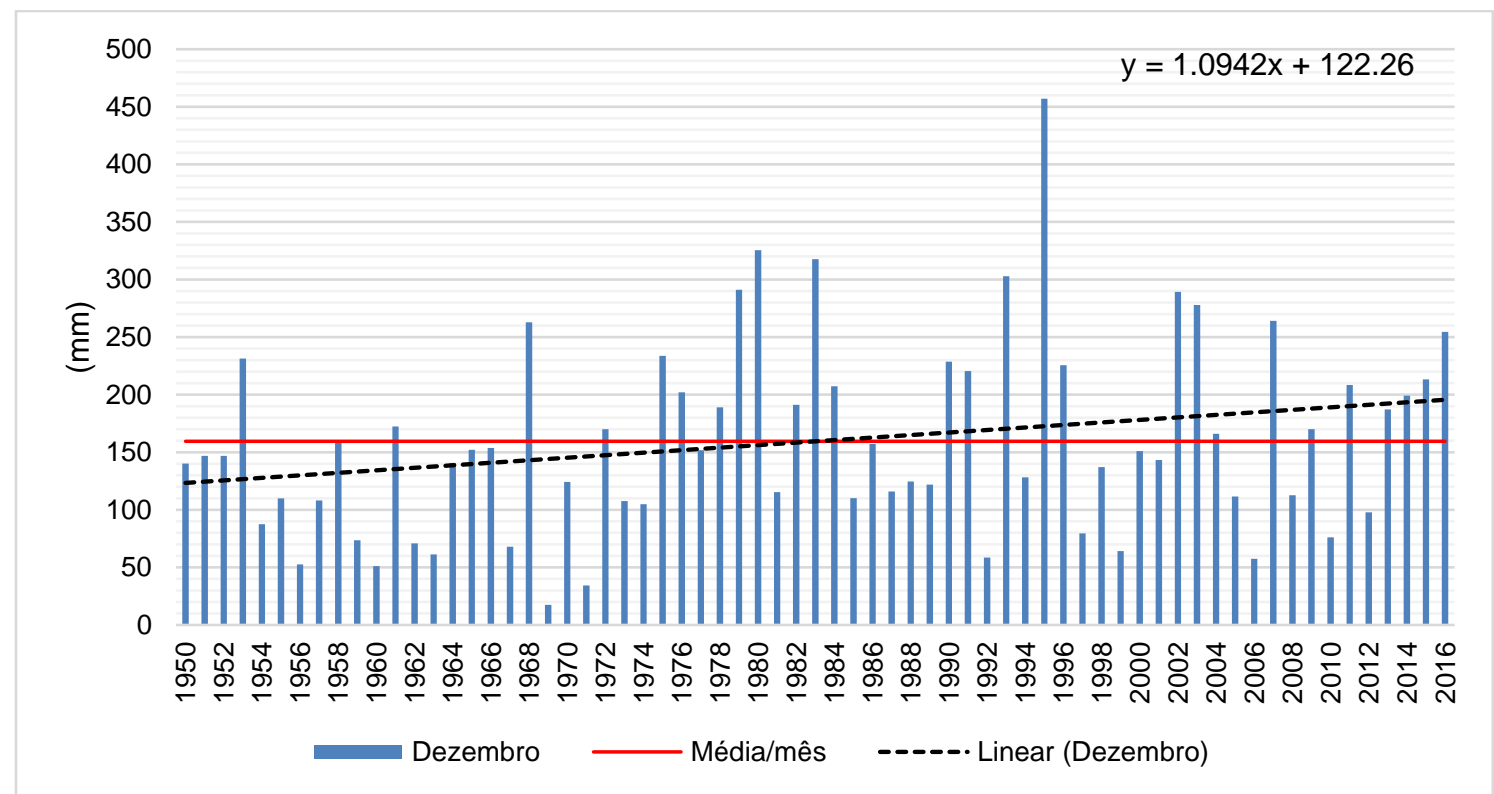

Fonte: Elaborado pelos autores (2018).

\subsection{ANÁLISES DE MANN-KENDALL}

A análise estatística de tendência de Mann-Kendall apresentou tendência positiva para aumento de chuvas nos meses de julho e dezembro, além dos totais anuais (Tabela 3). Os dados de dezembro e os anuais reforçam a análise de regressão, enfatizando tendência positiva para aumento de chuvas no sul catarinense, tomando por base os dados históricos. Observa-se que para o teste Mann-Kendall, na série anual, a tendência é significativa ( $\mathrm{p}=0,0002)$, sendo a magnitude $6,675 \mathrm{~mm}$ ano $^{-1}$, o que corresponde a aumento de $66,7 \mathrm{~mm}$ por década.

Tabela 3 - Análise de Mann-Kendall dos dados de chuvas (1950-2016)

\begin{tabular}{c|r|r|r|r|r}
\hline & \multicolumn{1}{|c|}{$\mathbf{Z}$} & \multicolumn{1}{c|}{ p-valor } & \multicolumn{1}{c|}{ Beta } & \multicolumn{1}{c}{ Beta inf } & \multicolumn{1}{c}{ Beta sup } \\
\hline Jan & 1,272 & 0,2034 & 0,8625 & $-0,3576$ & 2,2841 \\
\hline Fev & 1,261 & 0,2073 & 0,7417 & $-0,3849$ & 1,8462 \\
\hline Mar & 0,855 & 0,3926 & 0,3647 & $-0,5663$ & 1,2164 \\
\hline Abr & 0,498 & 0,6185 & 0,133 & 0,605 & 0,875 \\
\hline Maio & 1,596 & 0,1105 & 0,532 & $-0,1349$ & 1,223 \\
\hline Jun & 1,039 & 0,2988 & 0,2923 & $-0,2957$ & 0,8398 \\
\hline Jul & 2,32 & 0,02 & 0,73 & 0,11 & 1,3 \\
\hline Ago & 0,254 & 0,7995 & 0,0891 & $-0,6966$ & 1,0213 \\
\hline Set & 0,492 & 0,6227 & 0,1967 & $-0,6861$ & 1,1664 \\
\hline Out & 0,579 & 0,5626 & 0,2047 & $-0,5497$ & 1,0207 \\
\hline Nov & 1,25 & 0,2113 & 0,533 & $-0,35$ & 1,38 \\
\hline Dez & 2,1 & 0,0357 & 1,113 & 0,077 & 2,09 \\
\hline Ano & 3,723 & 0,0002 & 6,675 & 3,209 & 10,438 \\
\hline
\end{tabular}

Fonte: Elaborada pelos autores (2018). 
Quando se analisam os dados trimestrais, a estatística Mann-Kendall também reforça o $4 .^{\circ}$ trimestre como de tendência positiva de chuvas (Tabela 4), também coincidindo com os resultados já apresentados para regressão (Tabela 2).

Tabela 4 - Análise trimestral de Mann-Kendall (dados de chuva de 1950-2016)

\begin{tabular}{r|r|r|r|r|r}
\hline & \multicolumn{1}{|c|}{$\mathbf{Z}$} & \multicolumn{1}{c|}{ p-valor } & \multicolumn{1}{c|}{ Beta } & \multicolumn{1}{c}{ Beta inf } & \multicolumn{1}{c}{ Beta sup } \\
\hline $1^{\circ}$ trimestre & 1,753 & 0,0796 & 1,8515 & $-0,1864$ & 4,0163 \\
\hline $2^{\circ}$ trimestre & 1,396 & 0,1627 & 0,9556 & $-0,3253$ & 2,1838 \\
\hline $3^{\circ}$ trimestre & 1,699 & 0,0893 & 1,4219 & $-0,2278$ & 3,0304 \\
\hline $4^{\circ}$ trimestre & 2,24 & 0,0251 & 2,1061 & 0,3306 & 3,8191 \\
\hline
\end{tabular}

Fonte: Elaborada pelos autores (2018).

A título de exemplificação gráfica da análise de tendência (Figura 4), para o mês de dezembro veem-se os valores históricos, absolutos e independentes da série 1950-2016, em preto, bem como o ajuste da linha de tendência e sua previsão.

Figura 4 - Análise de tendência de Mann-Kendall para chuvas de dezembro (1950-2016) e previsão ascendente. Eixo $\mathrm{y}=$ precipitação e eixo $\mathrm{x}=$ tendência

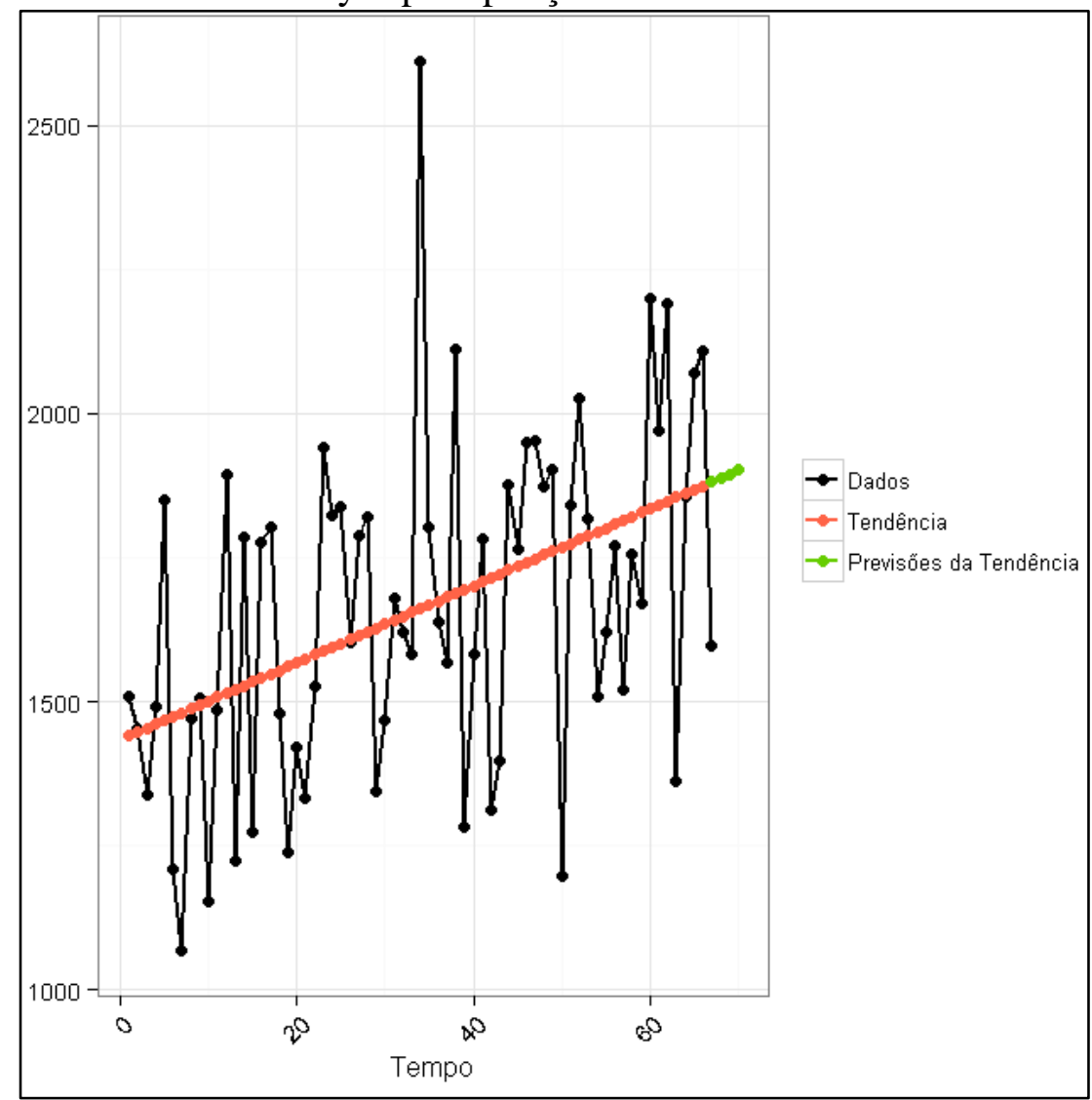

Fonte: Elaborada pelos autores (2018) 


\subsection{CHUVAS E ESTABILIDADE DE ENCOSTAS FLORESTADAS}

Os terrenos escarpados e declivosos do sul catarinense encontram-se, em sua maioria, vegetados por florestas. Segundo Neary et al. (2009), existe uma estreita relação entre solos florestais e eventos de precipitação extrema, uma vez que a elevada porosidade do substrato favorece a infiltração, reduz o escoamento superficial, a erosão, produz mais fluxo subterrâneo, "amortece" efeitos dos eventos extremos e favorece a melhoria da qualidade da água.

Quando a água da chuva atinge o solo da floresta, espera-se que a presença da vegetação florestal seja facilitadora da infiltração, para evitar que água escoe superficialmente e arraste sedimentos, detritos, ou mesmo desencadeie processos erosivos por escoamento hortoniano (NEARY et al., 2009). Assim, a quantidade de biomassa vegetal é inversamente proporcional à quantidade de chuva que se torna água superficial. Segundo os autores, em razão do escoamento hortoniano, o fluxo superficial ocorreria quase simultaneamente com a chuva, quando a quantidade precipitada excederia a de infiltração, sendo responsável pela rápida ascensão dos níveis dos rios, e causa responsável pela erosão (NEARY et al., 2009).

Observa-se que vários autores (HEWLETT; HIBBERT, 1963, TRIMBLE; WEIRICH, 1987; NEARY et al., 2009, HONDA; DURIGAN, 2017) convergem no entendimento de que, pela diminuição do escoamento superficial numa encosta, uma floresta tende a diminuir os processos erosivos, entretanto aumenta a infiltração e o conteúdo de água no solo, elevando a propensão à ocorrência de movimentos de massa. Rice et al. (1969) afirmaram que o escorregamento do solo seria resultado de uma falha de fricção ao longo de uma superfície de cisalhamento, essencialmente paralela à superfície topográfica (no caso dos escorregamentos translacionais), local esse em que as tensões cisalhantes acumuladas excedem as de resistência.

As condições mais favoráveis para escorregamentos ocorrem em declividades acentuadas, em solos sem coesão, como os coluviais, em períodos de forte chuva e sem proteção vegetal, contudo também ocorrem em áreas florestadas. As florestas facilitam a infiltração e diminuem o escoamento hortoniano, favorecendo a formação do solo, ou pedogênese. Kobiyama et al. (2012) alertam que, pela pedogênese, o solo aumenta em espessura e peso, e essa não é ilimitada, visto que há um momento em que o volume de material acumulado fica instável numa encosta, ou seja, é deflagrado o movimento de massa.

Conforme Kobiyama et al. (2012), o papel da floresta consiste em potencializar o escoamento subterrâneo em detrimento do superficial, modificando o balanço de um processo geomorfológico (erosão superficial) que se caracteriza por alta frequência e menores magnitudes, enquanto outro processo (escorregamento) apresenta maior magnitude e menor frequência. Entretanto, mais chuva afeta essa relação de frequência/magnitude, e mesmo encostas florestadas poderão escorregar. 


\section{CONSIDERAÇÕES}

A aplicação de modelo de regressão linear e análise de Mann-Kendall objetivou encontrar padrões de tendência positiva para os registros de chuva para o sul catarinense, que vem se mostrando com grande variabilidade e aumento de registros absolutos de precipitação. Essas mudanças convergem com a literatura sobre desastres e mudanças climáticas, uma vez que essas modificações atmosféricas em escala global estariam alterando os climas regionais.

Para o Estado de Santa Catarina, aponta-se como tendência o aumento dos índices de chuva, e essa foi confirmada com a análise de dados históricos da estação meteorológica de Urussanga, referência para o sul do Estado, numa série mensal de 1950 a 2016. O mês de dezembro, bem como o 4. ${ }^{\circ}$ trimestre do ano e a própria análise conjunta de todos os meses do ano, apresentou-se com tendência positiva de aumento de chuvas, e isso para as duas análises empregadas.

Essas informações permitem afirmar que há tendência de aumento no índice de chuvas para o sul catarinense, seja pontualmente para alguns meses, para último trimestre e, de fato, para o somatório dos anos analisados. Logo, o balanço hídrico nas encostas poderá ser alterado, e Santa Catarina possui um quadro fisiográfico com amplitudes altimétricas e declividades acentuadas.

Um solo saturado tende a aumentar a tensão cisalhante, ou seja, ficar sujeito a escorregamentos, aumentando a frequência de ocorrência desses. Mais chuva para o sul catarinense resultará em mais escorregamentos, que poderão ofertar um risco e classificarem-se em desastres. Adicionalmente, o planejamento das cidades e do campo, a exemplo de obras hidráulicas como canais, drenagens, abastecimento, dentre outros, deve ser pensado considerando-se essas tendências.

\section{REFERÊNCIAS}

AYOADE, J. O. Introdução à climatologia para os Trópicos. São Paulo: Difel. 1996.

BACK, A. J. Aplicação de análise estatística para identificação de tendências climáticas. Revista Pesquisa Agropecuária Brasileira, Brasília, v. 36, n. 5, p. 717-726, maio 2001.

BACK, A. J.; VIEIRA, H. J. Análise da tendência temporal nos dados de chuva de UrussangaSC. In: SIMPÓSIO INTERNACIONAL DE CLIMATOLOGIA, 3, 2009, Canela. III Simpósio Internacional de Climatologia. Sbmet, 2009. p.1-6.

BRASIL. $1^{\circ}$ Relatório de Monitoramento dos Indicadores Ambientais. Processo $\mathrm{n}^{\circ}$. 2000.72.04.002543-9. Justiça Federal. 1ª Vara Federal de Criciúma, SC. Criciúma, 2018. 308 p. CAMPOS, C.; ALVES, R.; BRAGA, H. J. Mudanças climáticas atuais e seus impactos no Estado de Santa Catarina. Agropecuária Catarinense. Florianópolis, v. 19, p. 31-35, 2006.

CEPED-UFSC - Centro Universitário de Estudos e Pesquisas sobre Desastres. Universidade Federal de Santa Catarina. Atlas Brasileiro de Desastres Naturais 1991 a 2012: volume Brasil. Florianópolis, 2013. Disponível em: <http://150.162.127.14:8080/atlas/Brasil\%20Rev\%202.pdf>. Acesso em: 30 jul. 2018. 
Revista Tecnologia e Ambiente, v. 25, 2019, Criciúma, Santa Catarina/SC ISSN Eletrônico 2358-9426 e ISSN Impresso 1413-8131

DENSKI, A. P. N.; BACK, A. J. Tendência climatológica nos elementos meteorológicos e na evapotranspiração de referência de Urussanga/SC. Revista Brasileira de Climatologia. Curitiba v. 17, n. 1, p. 262-275, jul./dez. 2015.

GOOSSENS, C.; BERGER, A. Annual and seasonal climatic variations over the northern hemisphere and Europe during the last century. Annales Geophysicae. Munich, v. 4, n. 4, p. 385-400, 1986.

HAYLOCK, M. R.; PETERSON, T. C.; ALVES, L. M.; AMBRIZZI, T.; ANUNCIAÇÃO, Y. M. T.; BAEZ, J.; BARROS, V. R.; BERLATO, M. A. M.; BIDEGAIN, M. A. M.; CORONEL, G.; CORRADI, V.; GARCIA, V. J.; GRIMM, A. M.; KAROLY, D.; MARENGO, J. A.; MARINO, M. B.; MONCUNILL, D. F.; NECHET, D.; QUINTANA, J.; REBELLO, E.; RUSTICUCCI, M.; SANTOS, J. L.; TREBEJO, I.; VINCENT, L. A. Trends in total and extreme South American rainfall 1960-2000 and links with sea surface temperature. Journal of Climate. Boston. v. 19, p. 1490-1512, 2006. Disponível em: <https://doi.org/10.1175/JCLI3695.1>. Acesso em: 4 fev. 2019. HELSEL, D. R.; HIRSCH, R. M. Statistical Methods in Water Resources. Techniques of WaterResources Investigations of the United States Geological Survey. Book 4, Hydrologic Analysis and Interpretation. USGS, 1991.

HELSEL, D. R.; HIRSCH R. M. Statistical Methods in Water Resources. U.S. Department of the Interior, 2002.

HEWLETT, J. D.; HIBBERT, A. R. Moisture and energy conditions within a sloping soil mass during drainage. Journal of Geophysical Research, Hoboken, v. 68, n. 4, p. 1081-1087, 1963. HIRSCH, R. M.; SLACK, J. R.; SMITH, R. A. Techniques of trend analysis for monthly water quality data: Water Resources Research. Hoboken, v. 18, n. 1, p. 107-121, 1982.

HONDA, E. A.; DURIGAN, G. A restauração de ecossistemas e a produção de água. Hoehnea. São Paulo v. 44, n. 3, p. 315-327, 2017.

IPCC - Intergovernmental Panel in Climate Change. Managing the Risks of Extreme Events and Disasters to Advance Climate Change Adaptation. Special Report of Working Groups I and II of the Intergovernmental Panel on Climate Change. Edited by C. B. Field et al.

Cambridge/New York, Cambridge University Press/IPCC, 2012.

IPCC - Intergovernmental Panel in Climate Change. Climate Change 2013: The Physical Science Basis. Contribution of Working Group I to the Fifth Assessment Report of the Intergovernmental Panel on Climate Change. Edited by T. F. Stocker et al. Cambridge/New York, Cambridge University Press, 2013.

IPCC - Intergovernmental Panel in Climate Change. Climate Change 2014: Impacts, Adaptation, and Vulnerability. Contribution of Working Group II to the Fifth Assessment Report of the Intergovernmental Panel on Climate Change. Edited by C. B. Field et al. Cambridge/New York, Cambridge University Press/

IPCC, 2014.

KOBIYAMA, M.; MICHEL, G. P.; GOERL, R. F. Relação entre desastres naturais e floresta.

Revista GeoNorte. Manaus, v. 6, p. 17-48, 2012.

MARENGO, J. M. O Futuro Clima do Brasil. Revista USP, São Paulo, n. 103, p. 25-32, 2014. MICHEL, G. P. Modelagem de estabilidade de encostas com consideração do efeito da vegetação. 2013. Dissertação (Mestrado em Engenharia Ambiental) - Programa de Pós-Graduação em Engenharia Ambiental, UFSC, Florianópolis, 2013. 152 p.

MINUZZI, R.B.; CARAMORI, P. H. Variabilidade climática sazonal e anual da chuva e veranicos no Estado do Paraná. Revista Ceres. Viçosa, v. 58, n. 5, p. 593-602, 2011.

MONTEIRO, M. A.; FURTADO, S. M. A. O clima no trecho de Florianópolis - Porto Alegre: uma abordagem dinâmica. Geosul. Florianópolis, v. 10, n. 19-20, p.117-133, 1996.

NEARY, D. G., ICE, G. G.; JACKSON, C. R. Linkages between forest soils and water quality and quantity. Forest Ecology and Management. Flagstaff, v. 258, p. 2269-2281, 2009. 
PEZENTE, D. P. Análise temporal do comportamento das chuvas na Bacia Carbonífera de Santa Catarina. In: V Congresso Brasileiro de Carvão Mineral. Criciúma. Anais... Criciúma: SATC, 29 maio/1 jun. 2017.

PINHEIRO, A.; GRACIANO, R. L. G.; SEVERO, D. L. Tendências das séries temporais de precipitação da Região Sul do Brasil. Revista Brasileira de Meteorologia. São José dos Campos, v. 28, n. 3, p. 281-290, 2013.

PORTAL ACTION. Teste de Mann-Kendall. Disponível em:

$<$ http://www.portalaction.com.br/series-temporais/223-teste-de-mann-kendall>. Acesso em: 2 ago. 2018.

REUBENS, B.; POESEN, J.; DANJON, F.; GEUDENS, G.; MUYS, B. The role of fine and coarse roots in shallow slope stability and soil erosion control with a focus on root system architecture: a review. Trees. Gent, v. 21, p. 385-402, 2007.

RICE, R. M., CROBETT, E. S.; BAILEY, R. G. Soil slips related to vegetation, topography, and soil in Southern California. Water Resources Research. Hoboken, v. 5, p. 647-659, 1969.

SELINUS, O.; ESBENSEN, K. Separating anthropogenic to natural anomalies in environmental geochemistry. Journal of Geochemical Exploration. Reston v. 50, p. 55-66, 1995.

SÔNEGO, M. O clima do litoral sul de Santa Catarina. In: Seminário Interdisciplinar dos Cursos de Licenciatura, 3. Criciúma. Anais... Criciúma: UNESC, p. 102-103, 2002.

TRIMBLE, S. W.; WEIRICH, F. H. Reforestation and the reduction of water yield on the Southern Piedmont since circa 1940. Water Resources Research. Hoboken, v. 23, p. 425-437, 1987. YU, P. S.; YANG, T. C.; CHOU, C. C. Effects of Climate Change on evapotranspiration from Paddy Fields in Southern Taiwan. Climatic Change, Dordrecht, v. 54, n. 1-2, p. 165-179, 2002. 